\title{
TWO-FOLD REGIONAL VARIATION IN INITIATION OF ANTI-OSTEOPOROSIS MEDICATION AFTER HIP FRACTURE IN THE UK
}

Anjali Shah ${ }^{1}$, Daniel Prieto-Alhambra ${ }^{1}$, Samuel Hawley ${ }^{1}$, Antonella Delmestri ${ }^{1}$, Janet Lippett ${ }^{2}$, Cyrus Cooper $^{1}$, Andrew Judge ${ }^{1}$, Kassim Javaid ${ }^{1}$

${ }^{1}$ University of Oxford, Oxford, UK, ${ }^{2}$ Royal Berkshire NHS Foundation Trust, Reading, UK

Background

About 88,000 hip fractures occur annually in the UK. Anti-osteoporosis drugs and other interventions to reduce falls can halve the risk of further hip fractures.

Objective

Describe the geographic variation in prescription of anti-osteoporosis drug therapy before and after a hip fracture during 1999-2013 in the UK.

Methods

We used primary care data (Clinical Practice Research Datalink) to identify patients with a hip fracture and primary care prescriptions of any anti-osteoporosis drugs (bisphosphonates, strontium, denosumab, oestrogen therapy, selective oestrogen receptor modulators, teriparatide) prior to the index hip fracture and up to 5 years after. Geographic variations in prescribing of generic oral bisphosphonates were analysed. Multivariable logistic regression models were adjusted for gender, age and body mass index (BMI).

Results

13,069 patients (mean age 82 years, $76 \%$ female) diagnosed with a hip fracture during 1999-2013 were identified. $11 \%$ had any anti-osteoporosis drug prescription in the 6 months prior to the index hip fracture. In the 0-4 months following a hip fracture $5 \%$ of patients were prescribed an antiosteoporosis drug in 1999, increasing to $51 \%$ in 2011 and decreased to $39 \%$ in 2013. In contrast there was little difference in the proportion of patients still on treatment at 5 years between 1999 and 2013 ( $15.9 \%$ vs. $15.8 \%$, respectively). The independent predictors of treatment initiation included men ( $\mathrm{OR}=0.4295 \% \mathrm{Cl}: 0.36-0.49$ ), increasing $\mathrm{BMI}(\mathrm{OR}=0.98$ per $\mathrm{kg} / \mathrm{m} 2$ 95\% $\mathrm{Cl}: 0.97-1.00)$ and geographic region $(\mathrm{OR}=1.2995 \% \mathrm{Cl}: 0.89-1.87$ North East region vs. $\mathrm{OR}=0.5695 \% \mathrm{Cl}: 0.43-0.73$ South Central region of the UK). The geographic differences in prescribing persisted over the 5 -year follow-up. If all patients were treated at the rate of the highest performing region, then nationally an additional 3,214 hip fracture patients would be initiated on therapy every year.

Discussion and Conclusion

Significant geographic differences exist in post-hip fracture prescribing of antiosteoporosis drugs despite adjustment for potential confounders at the patient level. While a significant increase was observed after 2005 , the rate of treatment initiation was still relatively low as was treatment rates at 5 years. Further work examining differences in health care provision may inform strategies to improve secondary fracture prevention after hip fracture. 